\title{
An image sensor with in-pixel selective-charge- subtraction circuits for selective light detection
}

\author{
Unghyun Kim ${ }^{\text {a) }}$ and Makoto Ikeda \\ Department of Electrical Engineering and Information Systems, \\ Graduate School of Engineering, The University of Tokyo, \\ 7-3-1 Hongo, Bunkyo-ku, Tokyo 113-8656, Japan
}

a)hyun@silicon.u-tokyo.ac.jp

Abstract: In this paper, a CMOS Image Sensor (CIS) capable for in-pixel selective light detection and background light suppression is presented. The pixel circuit is composed of demodulation transistors and Adaptive Charge Unit (ACU), realizes in-pixel selective-charge-subtraction. Demodulation transistors are used for selective light detection. And ACU is used for background light subtraction to prevent saturation for wide dynamic range. The presented image sensor has been fabricated in $0.18-\mu \mathrm{m}$ CMOS process and successfully tested, and it has a $64 \times 64$ pixel array. The minimum signalto-background ratio (SBR) is $-18.8 \mathrm{~dB}$ and the dynamic range is $89.3 \mathrm{~dB}$.

Keywords: modulated light, image sensor, background light suppression, light-section method, selective-signal detection

Classification: Integrated circuits

\section{References}

[1] M. Gupta, et al.: "Structured light in sunlight," IEEE International Conference on Computer Vision (ICCV) (2013) 545 (DOI: 10.1109/ICCV.2013.73).

[2] D. J.-H. Wu, et al.: "Triangulation-based laser range finder using a complementary metal-oxide semiconductor image sensor for application to automotive systems," Opt. Eng. 45 (2006) 044302 (DOI: 10.1117/1.2189831).

[3] J. Cheon, et al.: "Smart CMOS image sensor with high SBR and subpixel resolution for light-section-based range finding," IEEE Trans. Electron Devices 56 (2009) 2546 (DOI: 10.1109/TED.2009.2030708).

[4] Y. Oike, et al.: "A $120 \times 110$ position sensor with the capability of sensitive and selective light detection in wide dynamic range for robust active range finding," IEEE J. Solid-State Circuits 39 (2004) 246 (DOI: 10.1109/JSSC. 2003.820880).

[5] K. Yamamoto, et al.: "A $128 \times 128$ pixel complementary metal oxide semiconductor image sensor with an improved pixel architecture for detecting modulated light signals," Opt. Rev. 13 (2006) 64 (DOI: 10.1007/s10043-0060064-z). 
[7] L. Bonjour, et al:: "CMOS demodulation image sensor for nanosecond optical waveform analysis," IEEE Sensors J. 13 (2013) 1487 (DOI: 10.1109/JSEN. 2012.2237548).

[8] D. Stoppa, et al:: "A range image sensor based on 10-um lock-in pixels in 0.18um CMOS imaging technology," IEEE J. Solid-State Circuits 46 (2011) 248 (DOI: 10.1109/JSSC.2010.2085870).

[9] U. Kim and M. Ikeda: "A $64 \times 64$ image sensor with the capability of selective light detection and background suppression," IEEE International Conference of Sensors (2017) 1 (DOI: 10.1109/ICSENS.2017.8234364).

[10] M. Novak, et al.: "Visible light communication beacon system for internet of things," Conference on Microwave Techniques (2017) 1 (DOI: 10.1109/comite. 2017.7932304).

\section{Introduction}

Active sensing using image sensors are widely used in various applications, including 3-D range finding for mobile devices and automobiles. The projected and reflected light is often much weaker than the ambient light [1]. Therefore, the solutions against the strong ambient light are required for the image sensors. The conventional image sensors and position sensors can obtain the position of the projection light position of the three-dimensional measurement system from the position of the peak intensity of the sensor array plane [2,3]. Therefore, strong light projection is required for these sensors in real environment, such as outdoor environment with strong sunlight. A high-sensitivity image sensor with the capability of suppression of background light is required in this situation.

Correlation sensors are potential solutions to the difficulties. Different techniques have been used to design correlation sensors in the standard CMOS technology. Switched capacitor circuits are used to perform the charge transfers and demodulation functions at relatively low frequencies in [4]. However, because of the complicated circuits, the pixel fill factor of the sensor is low, the random noise effect is more significant, and the pixel requires high intensity of projected light to operate. Demodulation pixels in $[5,6]$ have also been constructed by using differential MOSFET pair mixers and integration capacitors. However, the mixer demodulator output depends significantly on the parasitic capacitance of photodiode. Some of others smart image sensor have been reported for detecting modulated light upto relatively high frequency [7]. However, due to the limited full well capacity, background light cannot be suppressed efficiently.

This paper presents a new approach for an image sensor that can detect the selective light while suppressing the background light for such application like 3 -D range-finding with modulated and coded light. For the detection of selectivesignal light from incident lights, a in-pixel demodulation transistors [8] are employed. And Adaptive Charge Unit (ACU) in pixel circuit is introduced to prevent the saturation problem by the background light. Demodulation transistors and a charge injection mode suppression circuit for background light realize high sensitivity. The image sensor has been designed, fabricated, and successfully measured. 


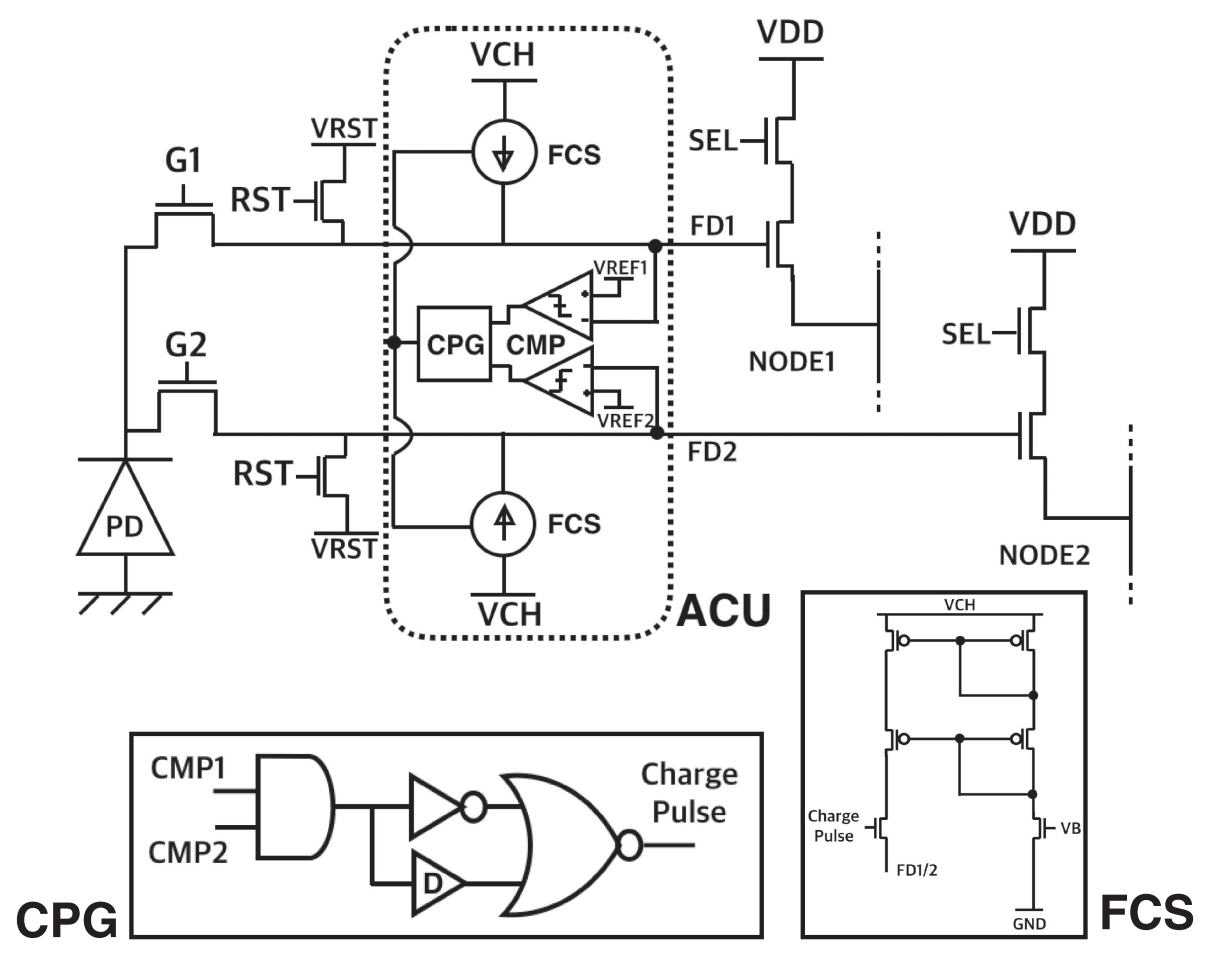

Fig. 1. Pixel circuit [9].

This paper is organized as follows: Section II introduces the target of rangefinding architecture based on structured light and triangulation. Section III describes the specification of our designed and fabricated image sensor and its array structure. Section IV explains the measurement results of our image sensor. Finally, conclusions are mentioned in Section V.

\section{Range-finding architecture}

The architecture of the proposed range-finding system [9] is described in this section. A new range-finding system, its pixel circuit with background suppression, and the principle of the pixel circuit are explained.

\subsection{New range-finding scheme}

New range-finding system [9] based on a light-section method using modulated light and an image sensor is proposed. In this system, the laser light source is modulated by a pulse generator. The modulated light sheet is projected onto the object. And the reflected light from the object go into the image sensor with the background light. The image sensor is controlled by a sampling frequency that is synchronized with the modulation frequency of the laser beam source. The sensor can sense only the reflected light from incident lights.

\subsection{Pixel circuit with background suppression}

Fig. 1 shows a schematic of the pixel circuit. The pixel circuit mainly consists of in-pixel demodulation transistors and Adaptive Charge Unit (ACU). ACU is employed to prevent the saturation problem in pixel during exposure time. In this pixel circuit, the difference of voltages between NODE1 and NODE2 means the 


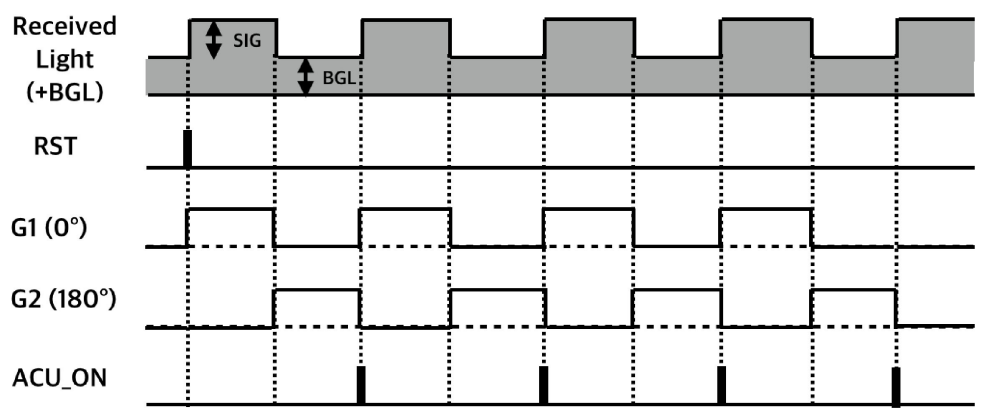

(a) Timing diagram of the sensing scheme.

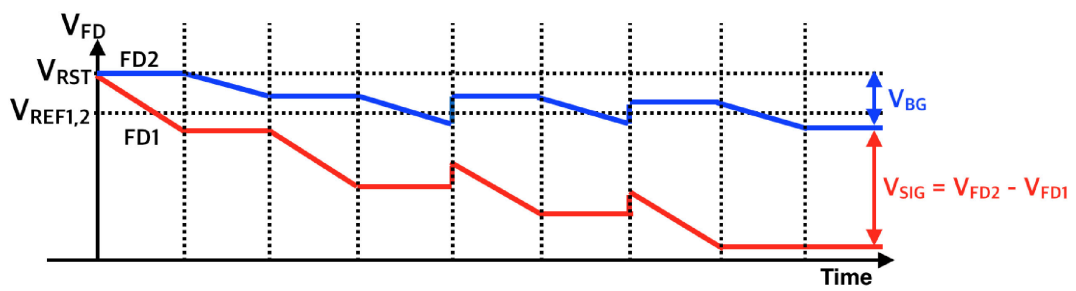

(b) Behavior of Active Charge Unite (ACU).

Fig. 2. Timing diagram of the sensing scheme and Behavior of Active Charge Unit (ACU).

value of the synchronization between light source and incident light. The bigger difference means more synchronized. After exposure, the 8bits digital data of the difference of the voltages between NODE1 and NODE2 are produced through readout circuit.

1) In-pixel demodulation transistors

As shown in Fig. 1, the Pixel has two gate transistor G1, G2. Fig. 2(a) shows a timing diagram of the sensing scheme. First, when the modulated light is $\mathrm{OFF}$, the reset operation is achieved by turning RST on. It is noted that the two reset transistors are connected each other, thus FD1 and FD2 are reset by this operation simultaneously. When the modulated light is turn on, G1 is turned on to transfer accumulated charges to FD1. Then the modulated light is turned off, and G1 is turned off and G2 is turned on to transfer accumulated charges to FD2. In ON-state of the modulated light, both modulated and background light components are stored in FD1. Next, in the OFF-state of the modulated light, only the static light component is stored in FD2. By repeating this operation, the charges in the ON- and OFF-states are accumulated in FD1 and FD2, respectively.

2) Active Charge Unit (ACU)

As illustrated in Fig. 2(b), Active Charge Unit (ACU) prevents the saturation problem by strong background light. During exposure time, ACU monitors the voltage levels of two nodes FD1 and FD2 and performs the charging operation into the nodes when its voltage levels of two nodes are in the specific condition. ACU consists of two comparators, Charge Pulse Generator (CPG), FD Charge Source (FCS).

- FD Level Detecting using comparators: During exposure time, the two comparators in pixel are monitoring by comparing the voltages of the 
two node FD1, FD2 with VREF1, VREF2 respectively. When the both voltages of the two nodes FD1 and FD2 are lower than the voltages of VREF1 and VREF2 respectively, the outputs of comparators turn on. While charging FD, when one of the voltages of the two nodes FD1 and FD2 is upper than the voltages of VREF1 or VREF2 respectively, the output of the comparator turns off.

- Charge Pulse Generator (CPG): A schematic of Charge Pulse Generator (CPG) is shown in Fig. 1. CPG makes the charge pulse from the output of the comparators. The charge pulse width is the period from turning the comparators on to off. Using the delay circuit, the charge pulse width can be fixed width. The delay circuit is consist of two inverters with bias transistors and can output ' 0 ' (default) or the delayed input (option) using the bias transistors.

- FD Charge Source (FCS): FD Charge Source (FCS) consists of cascade current mirror, switch and a bias transistor. A schematic of the FD Charge Source (FCS) is shown in Fig. 1. There are two FCSs in the pixel circuit. The outputs of FCSs are connected to two nodes FD1, FD2 respectively. The charge amount can be controlled by charge pulse width and the voltage $\mathrm{VCH}$ of FCS.

During exposure time, the ACU monitors the voltages of node FD1 and FD2 and operates charge-injection to the node FD1 and FD2 as below,

$$
\Delta V_{F D 1,2}=\frac{I_{\text {Charge }}}{C_{F D 1,2}} \times T_{\text {ChargePulseWidth }}
$$

Where, $I_{\text {Charge }}$ is the current from FCS (FD Charge Source) to nodes FD1 and FD2. $C_{F D 1,2}$ are constant value from pixel circuit. Then, $\Delta V_{F D 1,2}$ can be determined by only $T_{\text {ChargePulseWidth }}$.

\section{Chip implementation}

The proposed image sensor has been designed and fabricated in $0.18-\mu \mathrm{m}$ CMOS process. Fig. 3 shows a microphotograph of the fabricated image sensor and a layout of the pixel circuit. The pixel circuit occupies $40 \mathrm{um} \times 40 \mathrm{um}$ with $12.7 \%$ fill factor. The chip size is $5.18 \mathrm{~mm} \times 5.18 \mathrm{~mm}$.

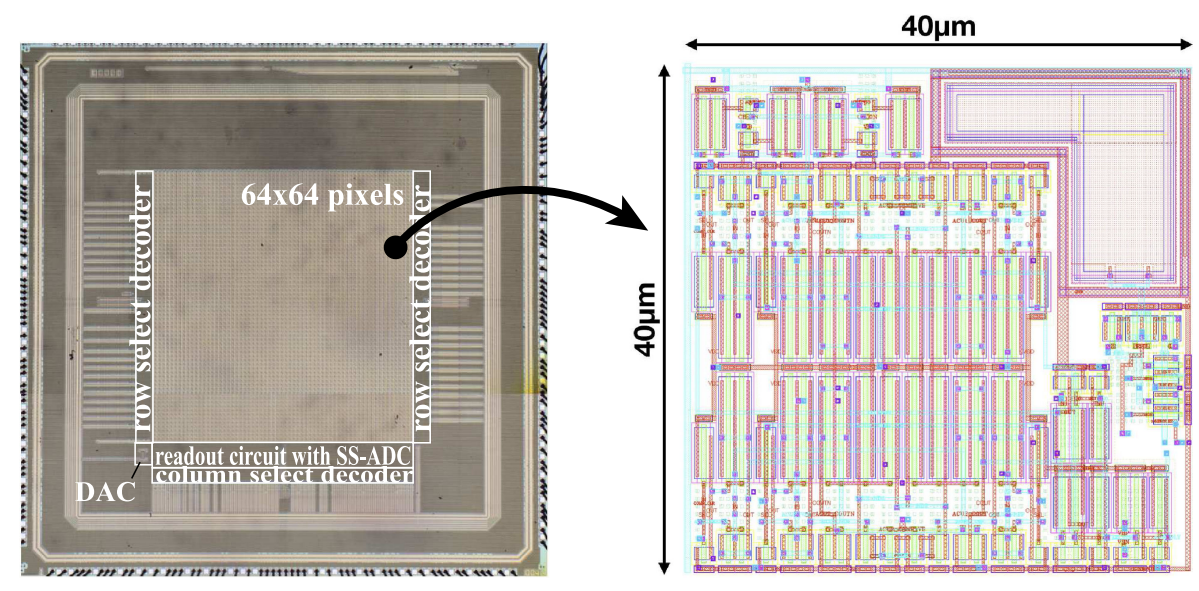

Fig. 3. Microphotograph of the fabricated chip and pixel layout. 


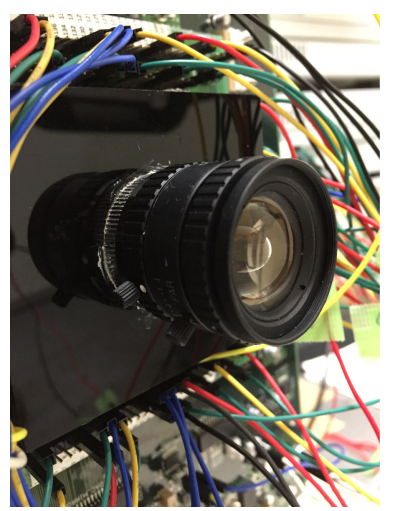

Fig. 4. Photograph of the sensor with lens on a test board

\subsection{Pixel array structure}

The image sensor consists of a $64 \times 64$ pixel array, a row-select address decoder, a column-select address decoder, a column-parallel readout circuit with Single-slope ADC, and a DAC. A specific pixel can be selected by the row-select and columnselect address decoder. After analog-to-digital conversion using column-parallel SS-ADC, its digitized output can be obtained from outside of chip.

\subsection{Readout circuit}

Readout circuit consists of a differential amplifier, single-slope ADC, 8-bit FFs and switches for readout to outside of the chip. At the differential amplifier, the difference voltage between NODE1 and NODE2 is amplified. The output of the differential amplifier is compared with the ramp signal from the DAC. When the output of amplifier overwhelms the ramp signal, the output of comparator turns ' 1 ' to ' 0 ' and the 8 -bits FFs latch the counter value at that time and keeps the value. After ADC process, the data in each column readout circuits are addressed to digital data bus outside of the chip in sequence.

\section{Measurement results}

The measurement system consists of a laser source (wavelength $670 \mathrm{~nm}$ ), the fabricated sensor with a lens mounted on a test board with FPGA as shown in Fig. 4, a pulse generator, a light projector for ambient illumination, and a lux meter. The sensitivity, the dynamic range, and the selectivity of the fabricated sensor are evaluated with the measurement system. In the procedure of post-processing for measurement, spatial low pass filter using $3 \times 3$ average filter is performed after accumulating and averaging 16 frames. The peak values are obtained by thresholding as shown in Fig. 5.

\subsection{Selective light detection}

Fig. 5 shows the ADC output from readout circuit at various incident light frequencies. In this measurement, the sampling frequency $F_{S}$ is $10 \mathrm{kHz}$ and the exposure time is 100 us. As shown by Fig. 5, only when the sampling frequency $F_{S}$ is equal to the frequency of the modulated light, the ADC output becomes a peak and the incident light with the other frequencies is suppressed. By using a set of laser frequencies such as 10,20, and $40 \mathrm{kHz}$, the proposed sensing scheme has high 


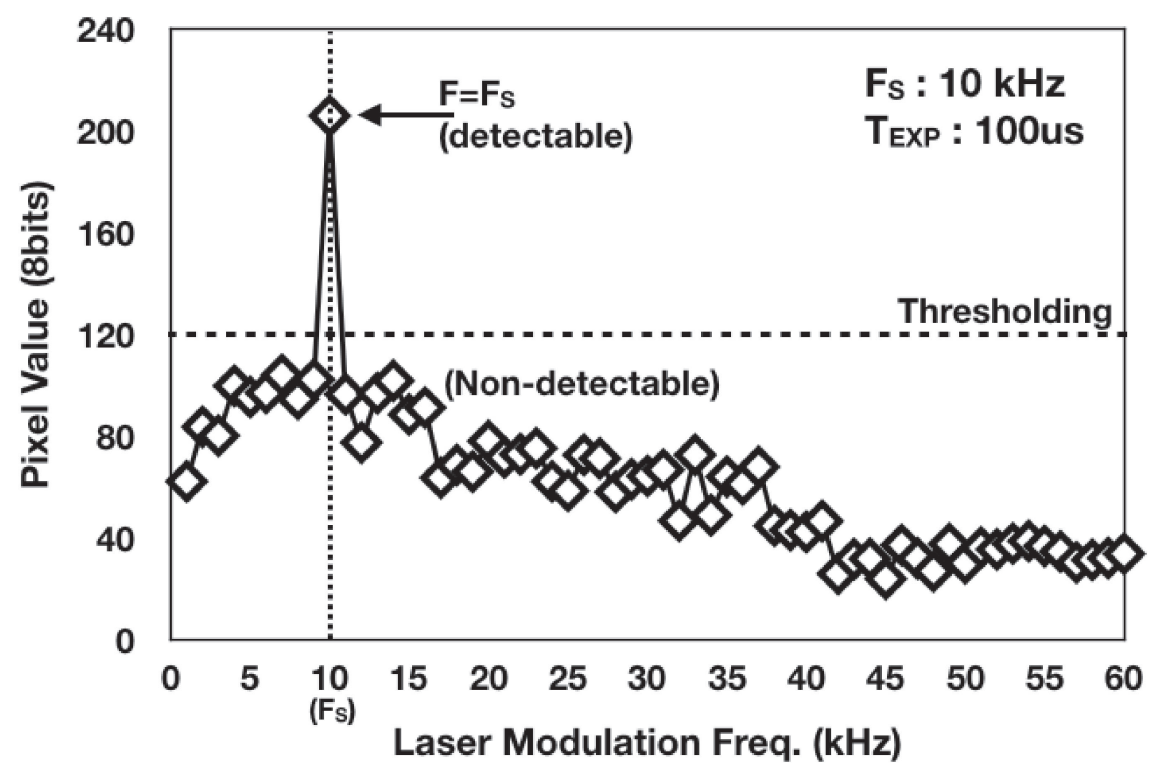

Fig. 5. Selective-Signal Detection.

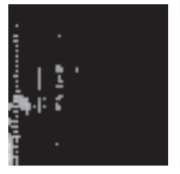

$F_{L}$ :

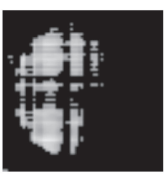

$10 \mathrm{kHz}$

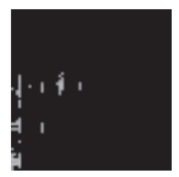

$20 \mathrm{kHz}$

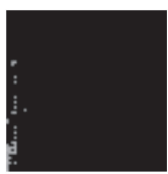

$30 \mathrm{kHz}$

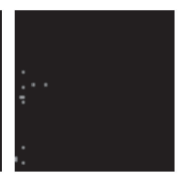

$40 \mathrm{kHz}$

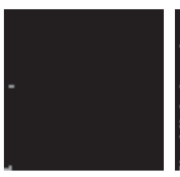

$50 \mathrm{kHz}$

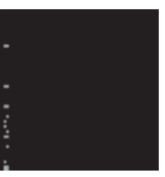

$60 \mathrm{kHz}$

Fig. 6. Output images when sampling frequency $F_{S}$ is $10 \mathrm{kHz}$ with various laser frequency $F_{L}$.

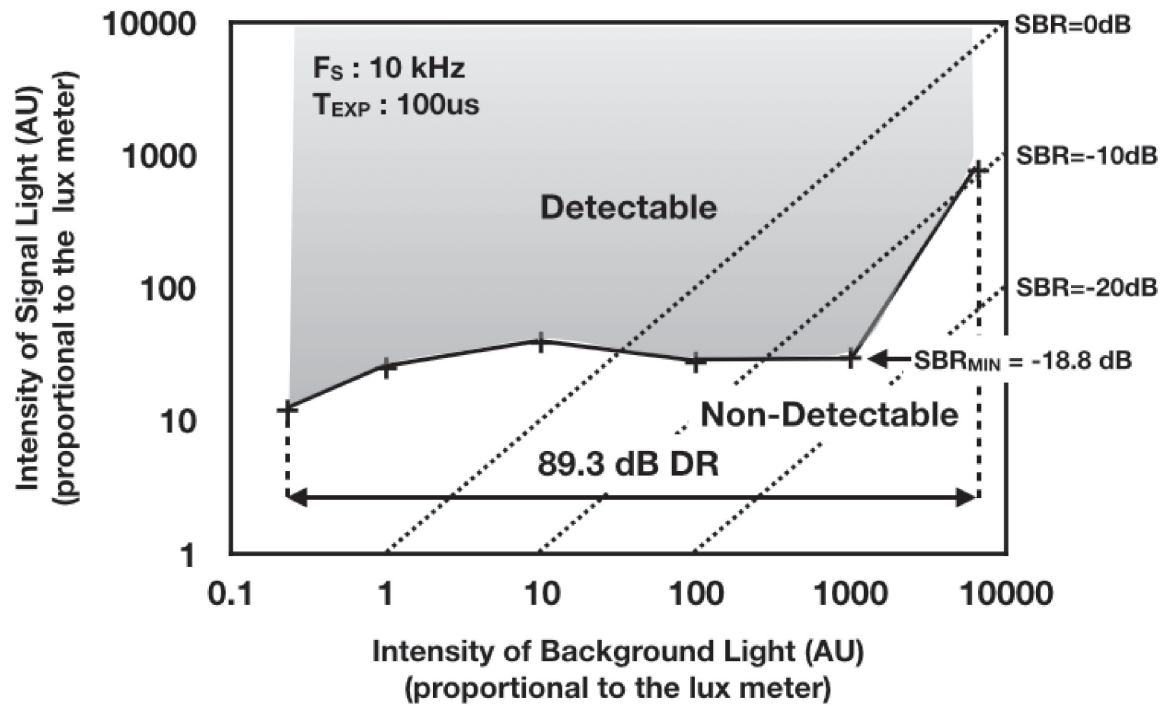

Fig. 7. Signal Background Ratio (SBR) and Dynamic Range.

sensitivity for a multiple light projection system. Fig. 6 shows the output images when sampling frequency $F_{S}$ is $10 \mathrm{kHz}$ with various laser frequency $F_{L}$ such as 5 , $10,20,30,40,50,60 \mathrm{kHz}$.

\subsection{Signal background ratio and dynamic range}

Fig. 7 shows the relationship between the minimum projected light intensity that can be detected and the intensity of background light. In the measurement of the 
Table I. Measurement results and comparison.

\begin{tabular}{|c|c|c|c|}
\hline Parameters & {$[4]$} & {$[5]$} & This Work \\
\hline $\begin{array}{c}\text { Buppression } \\
\text { Technique }\end{array}$ & $\begin{array}{c}\text { Current mode } \\
+ \text { correlation }\end{array}$ & $\begin{array}{c}\text { Separation } \\
\text { of AA* \& FD }\end{array}$ & $\begin{array}{c}\text { Charge } \\
\text { Injection }\end{array}$ \\
\hline SBR $(\mathrm{dB})$ & -22.8 & NA & -18.8 \\
\hline BGL DR $(\mathrm{dB})$ & 48 & 25 & 89.3 \\
\hline Pixel size $\left(\mu \mathrm{m}^{2}\right)$ & $60 \times 60$ & $30 \times 30$ & $40 \times 40$ \\
\hline Fill factor $(\%)$ & 13.5 & 26.2 & 12.7 \\
\hline $\begin{array}{c}\text { Maximum } \\
\text { modulation } \\
\text { frequency }(\mathrm{kHz})\end{array}$ & 10 & 0.5 & 60 \\
\hline
\end{tabular}

$\mathrm{AA}^{*}$ : accumulation area

signal-to-background ratio and dynamic range, the exposure time is 100 us. The sampling frequency $F_{S}$ and the modulation frequency are $10 \mathrm{kHz}$. For evaluation of the sensitivity of the image sensor, the intensities of the projected light and the background light are measured by lux meter. The minimum signal-to-background ratio (SBR) means the sensitivity of the image sensor. The Signal-to-background ratio (SBR) [3] is defined as below.

$$
S B R=20 \log \frac{I_{S I G_{M I N}}}{I_{B G}}
$$

As shown in Fig. 7, the present image sensor can detect the projected light that can be weaker than the background light. It means that the present image sensor can suppress the background light. The minimum signal-to-background ratio (SBR) is $-18.8 \mathrm{~dB}$. The dynamic range is $89.3 \mathrm{~dB}$.

\subsection{Comparison}

An overall comparison with the previous works is very difficult because of different parameters which are relevant depending on the application. By comparison with $[4,5]$ as shown in Table I, the present image sensor has less SBR than $-22.8 \mathrm{~dB}$ SBR reported in [4], but it achieves wider dynamic range than $[4,5]$. And it has a smaller pixel than $60 \mu \mathrm{m} \times 60 \mu \mathrm{m}$ in [4] and also has the maximum modulation frequency that is higher than $[4,5]$. The smaller pixel has benefit for high-resolution image sensor. In terms of the modulation frequency, there is a potential to be applied for some high-speed application such as visible light communication beacon system [10].

\section{Conclusion}

A new range-finding system, which is based on light-section method with the modulated light and the image sensor that can detect only the reflected light with the modulation by same frequency, has been proposed. Adaptive charge unit (ACU) in pixel circuit is employed to avoid saturation for wide dynamic range. The image sensor can detect the selective-signal light modulated by specific frequency. And the image sensor has been designed and measured. The minimum SBR is $-18.8 \mathrm{~dB}$ 
and the dynamic range is $89.3 \mathrm{~dB}$ in measurement. Our future works are the development and measurement for advanced three-dimensional measurement applications with this image sensor.

\section{Acknowledgments}

The image sensor in this study has been fabricated in the chip fabrication program of VLSI Design and Education Center (VDEC), the University of Tokyo in collaboration with Rohm Corporation and Toppan Printing Corporation. 